\title{
Smart Glove for Hearing-Impaired
}

\author{
Abhilasha C Chougule, Sanjeev S Sannakki, Vijay S Rajpurohit
}

\begin{abstract}
Individuals communicate with one another to pass on their thoughts to the general population around them. There are $2.78 \%$ of the total populations of India who can't speak. Gesture based communication is really a mode of correspondence for the general population who are either deaf or deaf-mute. Ordinary individuals don't become familiar with the gesture based communication. It causes conveyance gap between deafdumb and normal people. The past system of this project involved using image processing concept. But the downside of these past frameworks are projects were non portable and excessively costly. The aim behind this work is to build up a framework for perceiving the gesture based communication, which provides interaction between people who are deaf-dumb and normal people, thereby diminishing the interaction gap between them. Generally hearing-impaired people use linguistic communication based on hand gestures with specific movements to represent the ideas to others. The proposed glove is an robotic gadget that interprets American Sign Language Standard into text or speech in order to evacuate the information transmission gap between the mute and the ordinary public. This glove has been actualized with the assistance of flex sensors, accelerometer, microcontroller (Arduino Nano) and the Bluetooth chip.
\end{abstract}

Keywords- Flex sensor, Arduino Nano, MIT App inventor, Bluetooth chip, American Sign Language.

\section{INTRODUCTION}

Sign language may be a natural method of communication between traditional and deaf-dumb folks. Linguistic communication is usually enthusiastic about hand gesture recognition. A gesture is also outlined as a movement, sometimes of hand that expresses a thought. Linguistic communication may be an outlined method of conveyance within which each word or alphabet is delegated some gesture. It's generally difficult for traditional folks to acknowledge the signs properly and perceive what they require to mention. For instance, allow us to take into account a situation within which a traditional person desires to speak with an individual United Nations agency is hearing impaired and he's far from him, then the person cannot speak to him/her simply.

The planned work relies on a system which will perceive the linguistic communication accurately in order that the deaf-dumb folks may communicate with the final folks while not the requirement of associate interpreter. By implementing this planned system these disabled communities become freelance in life in order that they will even be a district of this growing digital world.

Revised Manuscript Received on April 12, 2019.

Abhilasha C Chougule, Department of Computer Science and Engieering, KLS'S Gogte Institute of Techology, Belgaum, India. (E-mail: chougule.abhilasha91@gmail.com)

Sanjeev S Sannakki, Department of Computer Science and Engieering, KLS'S Gogte Institute of Techology, Belgaum, India. (E-mail: sannakkisanjeev@gmail.com)

Vijay S Rajpurohit, Department of Computer Science and Engieering

KLS'S Gogte Institute of Techology, Belgaum, India. (E-mail: vijaysr2k@yahoo.com)
A gesture may be a specific movement of the hands with a selected form employed by the dumb folks to convey their thoughts to the general public. However most of the days they notice issue in human activity with others United Nations agency doesn't perceive linguistic communication. It's supported the requirement of developing associate device which will lower the gap or barrier between the mute folks and traditional society. There are several alternative sign languages accessible; they are American , British , Turkish, Indian Sign languages and plenty of a lot of.

Gesture recognition approach has a broad scope of utilizations and employments. This method can be utilized to control gadgets only via motions with no manual connection with the particular machine [7]. Using sign identification, an individual can pin out at the computer or at a versatile screen and utilize linguistic communication to pick and utilize totally distinctive implementations within the device. This linguistic communication employed by deaf and mute folks will be understood by the normal person this method and reborn to content so sanctioning and helping higher conversation among the deaf - mute and other individuals cooperating with them.

The Internet of things (IoT) is the internetworking of hand-to-hand contraptions, homes, autos and distinctive things embedded with hardware, sensors, actuators and device network that empower each thing to accumulate and trade information. The Iot lets in gadgets to be identified and controlled remotely across over current framework establishment, creating potential outcomes for too many direct combination of the physical worldwide into pc-based absolutely structure, and bringing about improved effectiveness, accurate and financial favorable position; while Iot is broadening with sensors and actuators, the age will turn into an occurrence of the additional well known style of digital real frameworks, which as well envelops previously comprehensive of brilliant networks, keen houses, insightful transportation and savvy towns. Each issue is particularly recognizable by means of its implanted registering gadget anyway is fit for interoperate inside the present web framework. Pros gauge that the iot will comprise of just about 50 billion contraptions by means of 2020.

The basic building squares of IoT are sensors and actuators. Sensors essentially sense the physical wonder that is happening around them; while actuators dependent on the detected data the actuators play out some activity on the physical prerequisites. 


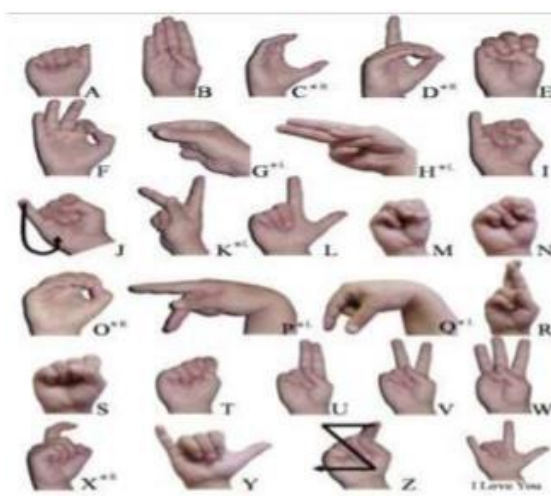

Fig 1. American Sign Language

\section{LITERATURE SURVEY}

According to the analysis done that, one out of every five people is deaf or dumb on this planet is an Indian. In India about more than 1.5 million deaf people utilize Sign Language [6] as a method of correspondence. Normal parents of deaf children or vice versa use gesture based conversation other than deaf population. However, due to this type of complications an automatic Sign-to- Speech/text language interpretation framework could assist to make more details accessible to the hearing impaired. In addition, the framework won't just promote data access; however it can likewise be utilized as an instructive apparatus to become familiar with any communication via gestures.

One of the earliest employments of a gesture based communication is from the 5th century BC, in Plato's Cratylus. In 1620, Juan Pablo Bonet proclaimed, Reduction of letters and art for instructing mute individuals to talk which is said to be the 1st present day investigation of communication via gestures vocal, mounting out a strategy for voice training for hard of deaf individuals and a stadard letter set.[1].

Thomas Pryor and Navid Azodi are UG understudies who made the Gloves that make an interpretation of communication via gestures into text and speech known as signaloud. They had won Lemelson-MIT understudy cost for this venture [3].The first Hand oration mittens was constructed by Ryan Patterson in the year 2001. This model had constraints that a PC or a workstation was constantly required for its working which made it less convenient and less portable.

In 2006, Nguyen Dang Binh et.al proposed "A New Approach Dedicated To Hand Gesture Recognition" utilized Thai communication via gestures acknowledgment with the strategy 14 ultra-data-glove which was fixed with 10 sensors for fingers and rest 4 sensors among the fingers which estimates variations and appropriation respectively. But, he got the 94\% outcome set. He utilized another new Pseudo 2D concealed markov model (P2DHMM) structure committed to the time series acknowledgment. In this procedure T-com P2DHMM structure was utilized to build up a total words of 36 gestures including the ASL letter spelling letters in order and digits and got $96 \%$ outcome set. [5]

"Hand Guesture Recognition System" Swapnil D. Badgujar, GourabTalukdar,Omkar Gondhalekar, Feb. 2014. Implemented by continuous gesture recognition a client can control a computer by making the gestures for which we want to convey using camera which is linked to the computer. [4]

"Signlanguage-speech conversion" Aarthi M, Vijaylakshmi P, 2016.Implemented a framework for changing over gesture based communication to speech utilizing LCD to show the communication through Sing language to text.

Cao dong et al. [2] utilized Microsoft kinect to recognize American Sign Language. Depth camera is utilized to distinguish ASL letters in order.

Analysis of the information with static images and recognize the image utilizing algorithms and produce sentences in the showcase, vision based communication through signing acknowledgment framework for the most part pursues the algorithms [8] are, used to extricate the pictures and dispense with the undesirable background tumult. The fundamental disadvantage of visualization in gesture based communication acknowledgment framework image acquisition operation has countless conservationist apprehensions, e.g. camera position, background disorder and brisk sensitivity. Camera site to focus the spot that acquire greatest feasible palm movements, steep resolution camera take up more reckoning time and possess more retention space. Camera is utilized all the time by clients but cannot actualize in open spot. Another exploration approach is a gesture based communication acknowledgment framework utilizing a data glove [9] [10].

\section{OBSERVATIONS}

\section{A. Sign language conversion using Android App.}

Android could be Linux-based software for mobile phones like sensible phones and computers. Android gives partner open stage to designers to make their own applications to be utilized by a scope of cell phones. An Android application for the framework was created utilizing Android Studio. HTTP communications protocol repository that produces connection for smart phone applications less demanding and speedier. It eases mechanized planning of meshwork appeals. In any convention it coordinates and offers help for raw strings, images, and JSON. The Android application can summon the camera that is in-built within the Android device. Pictures were then taken as input to Matlab for shape detection and classification.

For classification of shapes several applications make utilization of multivariate analysis, which relies on speculation that the cause and result association between the variables are ualtered. This may not perpetually be the case and thus approximation of the inconsistent values done on the idea of the regression of $\mathrm{y}$ on $\mathrm{x}$ might cause inaccurate outcomes. Back propagation rule is used for classifications. A giant and fluctuated dataset for instructing reason for the neural network area unit collected.

K Nearest Neighbors Classifier (k-NN or KNN) rule could be a methodology to perform the classification of objects supported knowledge that's distance learning nearest to the item [12].

Published By: 
Within the teaching part, the rule is just to reserve the vectors of options and classification of learning knowledge. Within the classification part, an equivalent options area unit calculated for the take a look at knowledge. The space of this new vector of all learning knowledge vector is evaluated, and therefore the variety of the nearest $\mathrm{k}$ taken. New purpose classification enclosed within the classification foreseen most of those points.

The linguistic communication device system has associate degree Android application. The clients can need to capture pictures by camera of the one who is creating the gestures using this app. These pictures can then be sent to the server and then send these pictures to MATLAB wherever gesture identification using neural network can ensue, which is trained to acknowledge ISL gestures. The photo is going to be depicted to the equivalent ISL gesture and reborn to text. The new texts are going to be redirected to Android application by a way of server and therefore the outputs are going to be displayed within the users Smartphone.

\section{B. Proposed Sign language conversion using gloves}

Smart glove is associate appliance that identifies the motions of the hand and fingers on an individual basis and sends these movements to the board within the variety of analog or digital pins. If outcomes of the system is in digital signals, are mapped to particular value for detecting each characters. These characters are then used in the formation of words and phrases. On this glove, different sensors are set to identify the gyro position of the hand. As a result using this glove hearing-impaired can convey ideas to the others easily.

So the significance of the gloves is to create the life kind of the dumb and deaf folks straightforward. the gloves interprets the hand gestures to text and any speech in order that the conventional folks will scan the recognized gesture and listen to the voice and perceive what that person desires to inform, which is able to create the communication more efficient. The system consists of each physical and nonphysical communication. Signing take issue from country to country it's not universally same. America developed American signing (ASL); British developed British signing and so on. The gloves convert the particular gestures to text and speech utilizing Arduino as heart of the system. The flex sensors are utilized in the system that is connected onto the gloves that convert the gesture into resistance that is any regenerate to the text through Arduino nano. The flex sensors come back from versatile sensors families that are versatile enough. Together with flex sensors measuring instrument and call sensors are used for correct output. The measuring instrument is employed to observe the motion of the hand and to observe the contact between the fingers that contact sensors are used. The choice of the sensors is predicated on the signs the language is consisting. some signs are enthusiastic about the movement of palm therefore to observe that measuring instrument is employed whereas therefore me signs are enthusiastic about the contact of the fingers so to urge the precise output if that signs contact sensors are used. The output of the sensors is processed on Arduino nano to urge text as associate output displayed on mobile screen. Any text transferred via Bluetooth module to mobile phones/computers. Any information regenerated to speech via text to speech conversion computer code. The analysis is being created to convert sign to speech and create it transportable, economical and extremely correct.

\section{METHODOLOGY \& RESULTS}

The overall functioning of the System is explained through the diagram shown in figure 2. It represents the final order and hierarchy of assorted operating blocks of the project. The person wears the glove that has flex sensors, accelerometer and measuring system seamed to that and makes the gesture per the American signing (ASL). Arduino Nano is employed to assemble signals from the flex sensors and accelerometer placed on the glove. Then the processed output is send over the MIT app to display the text output associated conjointly via a Bluetooth link to an Android Smartphone or a private laptop consisting of text to speech package (application) and speech output is obtained.

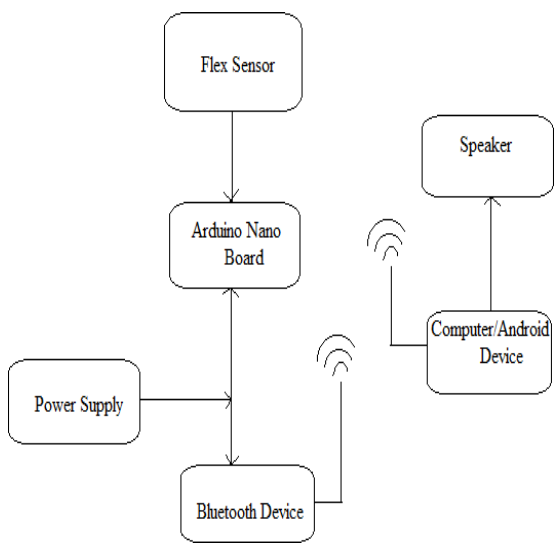

Fig 2. Block Diagram

The flow of algorithmic program employed in the system is as shown in figure 3 . The signing gloves are seamed with five flex sensors over the thumb, index, middle, ring, little fingers on the hand, Arduino nano and also the measuring system therefore acknowledge the precise movement of the fingers [13]. Every flex device is provided with the initial voltage as per the movement of the fingers there'll be a dip thanks to the modification within the resistance because of the bending of the flex device on the fingers. The voltages therefore obtained are analogous, which is then reborn to digital voltage mistreatment an analog to digital convertor using Arduino Nano. 


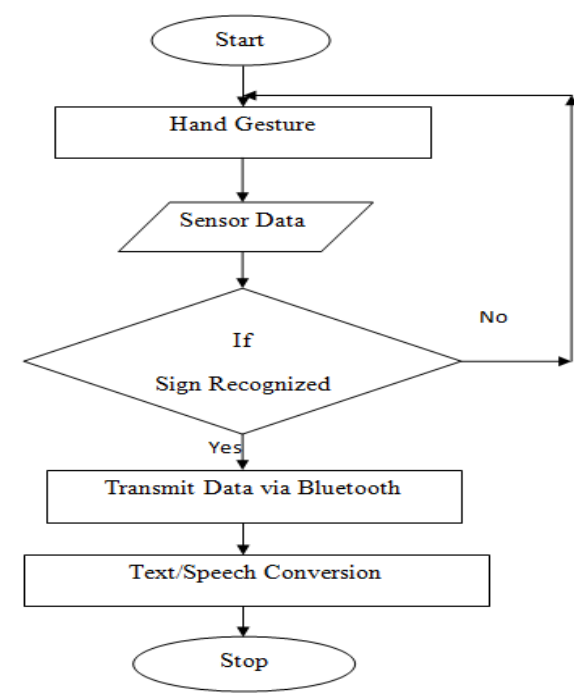

Fig 3. Flow Chart

The Digital output is then compared with the preloaded values of device within the system for the Alphabets and numbers, if the digital output matches the preloaded values then the popularity takes place of the Gestures and also the compared output is given as a text output on the mobile screen which can facilitate a standard person to browse and perceive. An equivalent output is transmitted over a Bluetooth module and so via Bluetooth link output is distributed to mechanical man Smartphone or a private laptop that is connected to the Bluetooth module and consisting of Text to Speech (TTS) conversion package (application) the serial input is received via the Bluetooth link on the connected device and a text output is received on the appliance with speech output from the speakers. This may facilitate traditional folks to browse the output and perceive the gesture alternatively hear the audio and communicate simply with the dumb and deaf folks [14]

\section{A. Flex Sensor}

Flex sensor means flexible sensors which change resistance based upon the curve made by fingers on the sensor. So the resistance is directly proportional to the bend made. [11]

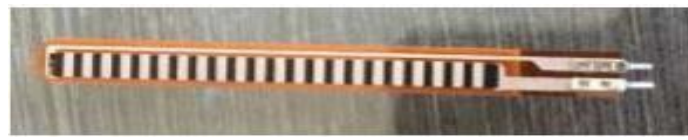

Fig 4. Flex Sensor

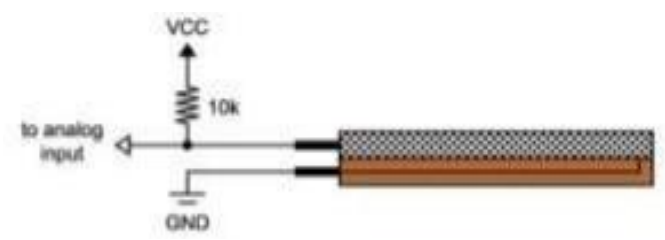

Fig 5. Voltage Driver (Flex sensor connection)

Flex sensor also work as variable analog voltage divider from figure 5. Flex sensor are made up of carbon resistive element within a thin bendable substrate as shown in figure 4. When the substrate is angled the resistive element gives a resistive output relative to the curve radius. The system consists of 5 flex sensors which are stitched on the fingers of the gloves because the main part of the gestures are fingers so for each finger a separate sensor is required. The hand gesture is inputted to the system via flex sensors the bent of each finger describes as shown in American Sign Language. As the fingers bends the sensors also starts bending and as the sensors bends the resistances also changes accordingly and that resistance value is inputted to the Arduino Nano.

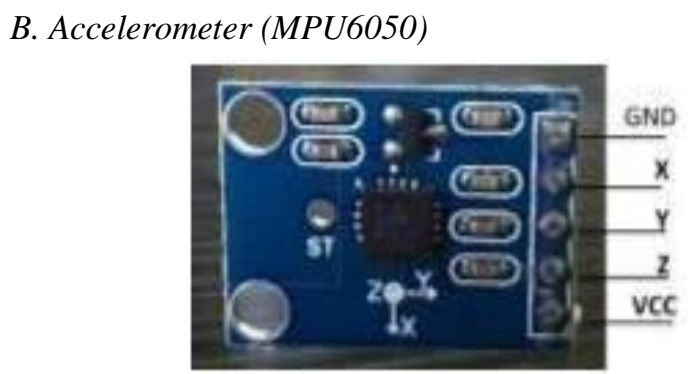

Fig 6 MPU6050

The accelerometer is a thin, small, low power. The accelerometer consists of 3 -axis $\mathrm{X}, \mathrm{Y}, \mathrm{Z}$ the change in position of the accelerometer changes the output of the $X$, $\mathrm{Y}$, and $\mathrm{Z}$ axis according to that output of the axis the gesture is recognized from figure 6. The sensor measures the gyro acceleration in lean-sensing applications and modern acceleration resulting from vibration. The accelerometer contains a crystalline surface semicromachined structure built on top of silicon wafer. The sensor measures the deflection of the surface and gives the deflection corresponding to particular axis. The accelerometer is placed on the wrist to sense the bending motion. Connection of accelerometer to the Arduino.

- Output pin connected to one of the analog pin on Arduino

- Vin pin is connected to 3.3V pin on Arduino

- GND pin is connected to ground pin on Arduino

\section{Bluetooth module}

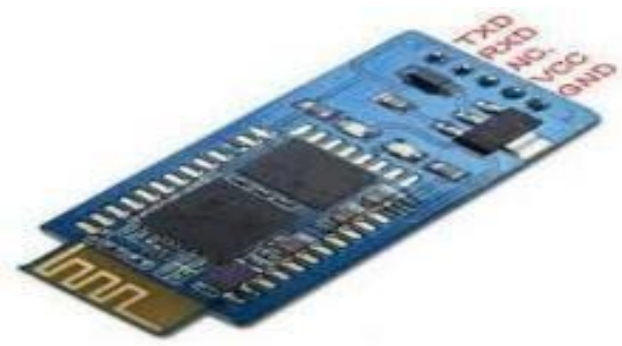

Fig 7. Serial port Bluetooth Module

The Bluetooth module is used to carry content from Arduino to mobile /computer shown in figure 7. This module sends serial data to the target device and the target device displays that data on the screen. The data sent to the Bluetooth module is the alphabets, numerical values, etc these data is then serially transferred to the target device and displays the output and further to software of speech conversion. Blue Eyes Intelligence Engineering 


\section{Arduino Software}

The Arduino IDE is a cross platform application written in java that utilizes the $\mathrm{C}$ programming language. This provides you access to a huge Arduino Library that's perpetually broadening because of open-source Company and in addition uploading programs to the board with one click can be done. First, we must always tack the board and port settings to allow us to transfer or upload code. Arduino boards are usually connected via the USB cable.

\section{E. Arduino Nano}

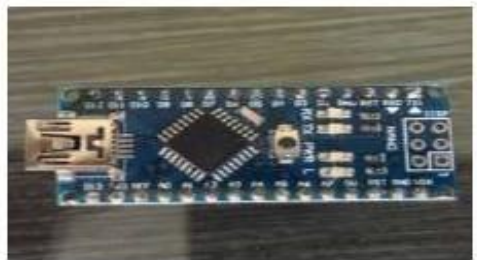

Fig. 8 Arduino Nano Board

The Arduino Nano is a small, complete, and breadboardfriendly board based on the ATmega328P, same as the Arduino UNO show in figure 8. The major didtinction between them is that the UNO board is presented with 30 pins and Nano is available with 32 pins. The extra 2 pins of Arduino Nano serve for the analog to digital conversion pins functionalities, while UNO and Nano has 6,8 ADC ports respectively. As other Arduino boards Nano board does not contain DC power jack, but instead has a mini-USB port that is used for both uploading programs and serial monitoring. One of the interesting features of Nano is that it will select the strongest power source with its potential difference, and the power source selecting jumper is invalid.

\section{F. MIT App Inventor}

It is an application which is used to prepare android applications by just dragging and dropping the buttons required for the development of applications. The text-tospeech block present in this software is used to convert the data to speak in mobile. In the inventor application all the components that an android phone consists are available like buttons, text boxes, etc, Sensors like accelerometer, barcode detector, location sensor, etc, social things like email, texting, twitter, etc, connectivity like Bluetooth, activity starter, web, etc. The app is used develop the application for text to speech conversion. In that application the device like android phone or computer is connected to the Bluetooth module of the device through Bluetooth connectivity of the device. After connecting to the system serial data is received and we get text to speech output.

\section{CONCLUSION}

Signal verbalization is one of the useful ways to ease the communication between the deaf and mute and normal society. Though signing may be enforced to speak, the target person should have a thought of the signing that isn't attainable forever. Thus it reduces such barriers. This paper was meant to be a typical example to visualize the feasibleness of recognizing sign languages. With this, deaf or mute communities will use the gloves to make gestures in keeping with signing and also the gestures are going to be converted to speech.
The system may be a bridge between traditional and dumbldeaf folks; it fills the gaps of communication between dumbldeaf folks and traditional people. The gloves area unit freelance and it's moveable with minimum weight and low power. The system converts the hand gestures to the text and more to speech. If in some conditions the person can't hear the sound produced there is a provision provided into the system of text due to which the person can read and understand what the another person wants to convey.

\section{REFERENCES}

1. Hussana Johar R.B, Priyanka A, Revathi Amrut M S, Suchitha K, Sumana K J "Multiple sign language translation into voice" International Journal of Engineering and Innovative Technology (IJEIT), Volume 3, Issue 10, April 2014.

2. Cao Dong, Ming C.Leu, Zhaozheng Yin, “ American sign language Alphabet Recognition Using Microsoft Kinect", Computer Vision and pattern Recognition workshop, IEEE conference, pp: ,2015.

3. http://lemelson.mit.edu/winners/thomas-pryor-and-navidazodi.

4. http://www.ijste.org/articles/IJSTEV2I9089.pdf

5. Nguyen Dang Binh, Toshiaki Ejima "A new Approach Dedicated to Hand Gesture Recognition" (Intelligence Media Laboratory, Kyushu Institute of Technology), Japan 2006 IEEE int. Conf. on cognitive informatics (iccie 06 ) Y.Y. Yao, Z.Z.Shi, Y.Wang, and W.kinsner (Eds.).

6. "Conversation of Sign Language to Speech with Human Gestures", ISBCC'2015 by Rajaganapathy. S, Aravind. B, Keerthana. B, Sivagami.M.

7. Wen Gao and GaolinFanga,"A Chinese sign language recognition system based on SOFM/SRN/HMM. Journal of Pattern Recognition".2389-2402,2004.

8. Nicholas Born.,"Senior Project Sign Language Glove", electrical engineering department. California Polytechnic State University, 1- 49,2010

9. Kirsten Ellis and Jan Carlo Barca."Exploring Sensor Gloves for Teaching Children Sign Language. Advances in HumanComputer Interaction".1-8.,2012

10. SolankiKumar "“ Indian Sign Language using Flex sensor Glove" International Journal of Engineering Trends and Technology(IJETT)vol.4,n0.6 June 2013.

11. Jinho Kim, Byung-Soo Kim, Silvio Savarese Comparing Image Classification Methods: K-Nearest-Neighbor and Support- VectorMachines.

12. "Translating Indian Sign Language to text and voice messages using flex sensors", International Journal of Advanced Research in Computer and Communication Engineering Vol 4, Issue 5, May 2015 by Sachin Bhat, Amruthesh M, Ashik, Chidanand Das, Sujith.

13. "Hand Gesture Recognition to Speech Conversion in Regional Language", International Journal of Computer Science and Network, Volume 4, Issue 1, February 2015 by B.D.Jadhav, 2 Nipun Munot, 3 Madhura Hambarde, 4 Jueli Ashtikar JSPM's Rajarshi Shahu College of Engineering, Pune, India.

14. "Sign Language To Speech Conversion Using Arduino" ,International Journal of Recent Innovation in Engineering and Research Scientific Journal Impact Factor - 3.605 by Yash Jhunjhunwala1, Pooja Shah2, Pradnya Patil3 and Jyot Waykule Sou. Sushila Danchand Ghodawat Charitable Trust's Sanjay Ghodawat group of Institutions. 